\title{
Health-Related Quality of Life and the Relationship to Treatment Satisfaction in Patients with Multiple Sclerosis: Insights from a Large Observational Study
}

This article was published in the following Dove Press journal: Patient Preference and Adherence

\author{
Dirk Schriefer (D) \\ Rocco Haase (iD) ${ }^{\prime}$ \\ Jennifer S Kullmann ${ }^{2}$ \\ Tjalf Ziemssen (D) \\ 'University Clinic Carl Gustav Carus \\ Dresden, Center of Clinical \\ Neuroscience, Dresden, Germany; \\ ${ }^{2}$ Sanofi-Aventis Deutschland $\mathrm{GmbH}$, \\ Neu-Isenburg, Germany
}

Introduction: In patients with multiple sclerosis (MS), fatigue, depression, and physical disability are important determinants that negatively affect health-related quality of life (HRQoL). In studies about MS, HRQoL and treatment satisfaction are emerging endpoints representing the patients' perspective. However, the association of HRQoL and MS treatment satisfaction has not been evaluated so far.

Purpose: Our objective was to evaluate the relationship of different dimensions of HRQoL and treatment satisfaction (effectiveness, side effects, convenience), and to assess which factors of treatment satisfaction, besides disease-related and sociodemographic explanatory factors, can best describe HRQoL.

Patients and Methods: We analyzed data from a cross-sectional, observational multicenter study in Germany (THEPA-MS, N=2990 eligible patients for first-line treatment). The instruments used were the SF-36 for HRQoL and the TSQM for treatment satisfaction. Correlation analyses, classification and regression trees and multivariate linear regression with the least absolute shrinkage and selection operator (LASSO) for global variable selection were used to analyze explanatory factors of HRQoL.

Results: The SF-36 physical summary score was $45.49 \pm 12.03$ and mental component summary score $42.87 \pm 12.12$, with currently untreated patients $(\mathrm{N}=250)$ reporting lower HRQoL than patients under first-line treatment $(\mathrm{N}=2740)(\mathrm{p}<0.001)$. Physical disability (standardized beta $(b)=0.408$ ) was the strongest cross-sectional predictor for physical health, followed by employment status $(b=0.163)$, age $(b=0.159)$ and treatment satisfaction in terms of side effects $(b=0.146)$ and effectiveness $(b=0.137)$. For the mental summary health dimension, presence of a major depressive episode $(b=0.234)$ had the greatest impact, followed by satisfaction with side effects $(b=0.152)$ and effectiveness $(b=0.131)$.

Conclusion: Satisfaction with the effectiveness and side effects of treatment was part of the main independent explanatory variables for mental and physical HRQoL in patients with MS. To improve HRQoL, patients' needs and satisfaction measures may be integral part of disease management beyond treatment of physical disability, depression or fatigue.

Keywords: health-related quality of life, treatment satisfaction, patient-reported outcome, TSQM, SF-36, multiple sclerosis

\section{Introduction}

Multiple sclerosis (MS) is a chronic inflammatory progressive disease of the central nervous system manifested by demyelination and axonal degeneration, and is known to worsen over time. ${ }^{1}$ First-line treatment options aim primarily at reducing the relapse rate and slowing down the progression of the disease. However, the
Correspondence: Tjalf Ziemssen Tel +49-35I-458-4465

Fax +49-35I-458-57I7

Email tjalf.ziemssen@uniklinikumdresden.de 
etiology of MS remains unclear and individual disease progression highly variable. ${ }^{2}$ As a chronic and incurable disease with a demanding lifelong treatment regimen, MS significantly impairs health-related quality of life (HRQoL). ${ }^{3}$

HRQoL can be conceptualized as those areas of quality of life (QoL) which are affected by health status while general QoL often includes economic, cultural and social environment aspects. HRQoL dimensions are measurable on an individual level, for instance as physical and mental health. ${ }^{3,4}$

In addition to HRQoL, the concept of treatment satisfaction is frequently used in clinical research to capture more immediate treatment-related experiences for patients, especially in terms of perceived medication effectiveness, side effects of treatment, or convenience of use. ${ }^{5,6}$

HRQoL and treatment satisfaction are commonly assessed as patient-reported outcomes (PROs). Fatigue, depression, physical disability and cognition are important determinants of reduced HRQoL in patients with MS..$^{7-9}$ Factors that are significantly related to patients' satisfaction with treatment have also been widely investigated. ${ }^{10,11}$ For both HRQoL and treatment satisfaction, there is evidence for a direct or indirect association to MS treatment adherence. ${ }^{6,12-14}$ Adherence to treatment in turn may promote reduced relapse rates, slow disability progression, and therefore stabilize longterm MS outcomes. ${ }^{15}$

An increasing number of clinical trials and real-world studies in MS use HRQoL and treatment satisfaction as primary or secondary outcomes. ${ }^{16,17}$ However, to our knowledge, the relationship between different dimensions of HRQoL and treatment satisfaction has not been reported in detail before.

\section{Objectives}

The primary goal was to examine HRQoL in a large cohort of MS patients treated with first-line disease-modifying therapies (DMT) and to investigate the relationship with treatment satisfaction. Particularly, we quantified the cross-sectional predictive value of treatment satisfaction in terms of side effects, convenience and effectiveness for both the physical and mental health dimensions of HRQoL by means of a comprehensive multivariate analysis of approximately 3000 patients under clinical practice conditions.

\section{Patients and Methods}

The non-interventional study "Therapy satisfaction in patients with relapsing-remitting multiple sclerosis"
(THEPA-MS) study was a multicenter, observational, crosssectional study on patients treated with first-line therapies. It was conducted between August 2013 and April 2014 and 445 neurological practices across Germany participated. During this period, patients were consecutively documented at a single time point by their attending neurologist and filled in validated questionnaires on $\mathrm{HRQoL}$ and treatment satisfaction during this occasion.

\section{Ethics and Patient Consent}

This study complies with the World Medical Association's Declaration of Helsinki. Written informed consent was obtained from all patients prior to inclusion. The study materials (observational plan, case report form, patient questionnaires, patient information sheet, informed consent form) were approved by the ethics committee of the Medical Faculty of the Technical University of Dresden.

\section{Inclusion Criteria}

Patients were eligible if they had a verified MS diagnosis according to the McDonald criteria. ${ }^{18}$ Inclusion criteria were: age of 18 years and older; clinically isolated syndrome (CIS) or relapsing-remitting MS type (RRMS); MS first-line therapy at the time of documentation as recommended by the guidelines released by the German Neurological Society for both CIS and RRMS patients: ${ }^{19}$ interferon (IFN) beta-1a intramuscular (i.m.), IFN beta-1a subcutaneous (s.c.), IFN beta-1b s.c., glatiramer acetate (GA); or at least principally eligible for first-line treatment; never had an escalation therapy. For the analysis reported here, treatment-naive patients were excluded as they were not able to evaluate treatment satisfaction in a valid way.

\section{Measures}

For each patient, physicians documented sociodemographic characteristics and therapy in detail.

MS and therapy data collected were: date of diagnosis, current MS diagnosis, current Expanded Disability Status Scale (EDSS) score, ${ }^{20}$ presence of fatigue, presence of a major depressive disorder (depression), relapses within the last year prior to the documentation date, current MS basic therapy (DMT type) and number of treatment changes. In addition, reasons for the discontinuation of therapy were documented for currently untreated patients.

Patients filled in questionnaires on HRQoL (Medical Outcome Study Health Survey 36-Item Short Form version 2.0, SF-36) and treatment satisfaction (Treatment Satisfaction Questionnaire for Medication Version 1.4, 
TSQM). ${ }^{21-23}$ The SF-36 provides a subjective measure of health status across eight scales: physical functioning (PF: ten items), role participation with physical health problems (RF: four items), bodily pain (BP: two items), general health (GH: five items), vitality (VT: four items), social functioning (SF: two items), role participation with emotional health problems (RE: three items) and mental health (MH: five items). All health domain scales were scored from 0 to 100, the higher the score the higher the HRQoL. For reduction of dimensionality, two norm-based summary scores were derived, the physical component summary score (PCS) and the mental component summary score (MCS). ${ }^{21}$

The TSQM was developed as a general measure of patient satisfaction with their current medication. In addition to global treatment satisfaction ( 3 items), satisfaction is also evaluated in the dimensions of side effects (5 items), effectiveness ( 3 items) and convenience ( 3 items). TSQM domain scores range from 0 to 100 , with higher scores representing higher treatment satisfaction. The TSQM has shown good psychometric measurement characteristics in terms of reliability and validity in patients with various chronic diseases, ${ }^{23}$ including patients with $\mathrm{MS}^{24}$

\section{Statistical Procedure}

For comparisons between treatment groups, ANOVA, Student's $t$-test, Kruskal-Wallis $H$-test, Mann-Whitney $U$ or Chi-squared tests were used, as appropriate. For ANOVAbased analyses, eta squared was additionally reported as a measure of effect size and thresholds of $0.01,0.06$ and 0.14 were considered small, medium and large, respectively. ${ }^{25}$ For interference on reference data from a healthy German reference population, bootstrapping resampling method with bias-corrected and accelerated $(\mathrm{BCa})$ confidence intervals was applied for the single HRQoL domains (PF, RF, BP, GH, VT, SF, RE, MH) and Student's $t$-test for the summary measures (PCS, MCS). ${ }^{26}$ Spearman rank correlations were calculated to study bivariate relations of the SF-36 domains and TSQM dimensions. Correlations of 0.10, 0.30 and 0.50 were considered as relatively small, medium and relatively large effect sizes, respectively. ${ }^{25,27}$

Decision trees were constructed using Exhaustive Chisquared Automatic Interaction Detection (Exhaustive CHAID) to quantify the connection of all explanatory variables on each HRQoL outcome (PCS, MCS, PF, RF, BP, GH, VT, SF, RE, MH). Exhaustive CHAID is a data-driven, nonparametric tree technique based upon Bonferroni adjusted significance testing with automatic regrouping of all predictors (recursive partitioning). ${ }^{28}$

To investigate the factors that potentially affect and may best cross-sectionally predict HRQoL on a global level, two regression models were constructed using PCS and MCS as dependent variables. For variable selection, the Least Absolute Shrinkage Selection Operator (LASSO) was implemented through the categorical regression procedure. ${ }^{29,30}$ LASSO is a shrinkage technique and proposed as a tool of automatic variable selection overcoming limitations from classical stepwise procedures. ${ }^{31}$ Finally, multivariate linear regression models (without shrinkage) were implemented with the set of predictors which were derived from the LASSO. Corresponding standardized beta coefficients were reported as effect sizes to compare the relative importance of the explanatory variables.

Statistical significance was predefined on the $5 \%$ level and all comparisons were carried out two-tailed. No imputation techniques were implemented, but in tree analyses, missing values were included as separate categories. All statistical analyses were performed using IBM SPSS version 25.0 (IBM Corporation, Armonk, NY, USA).

\section{Results}

\section{Characteristics}

Overall, 3312 patients were enrolled and characteristics of 3181 eligible patients have been published before. ${ }^{11}$ After exclusion of 191 treatment-naive patients, 2990 patients remained for the final analysis set.

A total of 2831 patients $(94.7 \%)$ presented a relapsingremitting course and 159 (5.3\%) had CIS (Table 1). Mean patient age was $43.36 \pm 11.09$ years at the time of documentation and approximately three-quarters were female (Table 1). Average disease duration was $8.78 \pm 6.71$ years (median 7.0). Mean EDSS was $2.26 \pm 1.52$ (median 2.0) and $37.5 \%$ of the patients faced at least one relapse in the year before study entry. MS-related fatigue was documented in $45.5 \%$ and a clinical depression in $16.9 \%$ of cases. Overall, $250(8.4 \%)$ patients were currently untreated but had received first-line therapy in the past. A total of 2740 patients $(91.6 \%)$ received any type of IFNbeta or GA basic therapy (IFN/GA): 704 patients (23.5\%) IFN-beta 1a i.m, 687 patients (23.0\%) IFN-beta 1a s.c., 564 patients (18.9\%) IFN-beta 1 b s.c and 785 (26.2\%) patients were treated with GA. Nearly three-quarters of all patients never had a change in their DMT history (Table 1). 
Table I Patient Characteristics (N=2990)

\begin{tabular}{|c|c|c|c|}
\hline & & Percent & $(95 \% \mathrm{Cl})$ \\
\hline Gender & Female & $73.3 \%$ & $(71.72-74.92)$ \\
\hline Employment & Not Working & $32.5 \%$ & $(30.79-34.22)$ \\
\hline Living with a partner & No & $23.3 \%$ & $(21.78-24.96)$ \\
\hline \multirow[t]{2}{*}{ Disease course } & $\mathrm{ClS}$ & $5.3 \%$ & $(4.54-6.18)$ \\
\hline & RRMS & $94.7 \%$ & $(93.82-95.46)$ \\
\hline Fatigue & Yes & $45.5 \%$ & $(43.74-47.37)$ \\
\hline Depression & Yes & $16.9 \%$ & $(15.55-18.30)$ \\
\hline Relapses & One or more & $37.5 \%$ & $(35.74-39.25)$ \\
\hline \multirow{2}{*}{$\begin{array}{l}\text { Current disease-modifying } \\
\text { therapy }\end{array}$} & Yes (IFN/GA) & $91.6 \%$ & $(73.93-94.11)$ \\
\hline & No DMT & $8.4 \%$ & $(05.89-26.07)$ \\
\hline \multirow{3}{*}{$\begin{array}{l}\text { Duration of medication } \\
\text { intake }\end{array}$} & $<2$ years & $29.1 \%$ & $(27.48-30.80)$ \\
\hline & $2-8$ years & $44.8 \%$ & $(42.94-46.57)$ \\
\hline & $>8$ years & $26.1 \%$ & $(24.55-27.76)$ \\
\hline \multirow{3}{*}{$\begin{array}{l}\text { Number of medication } \\
\text { changes }\end{array}$} & No changes & $71.8 \%$ & (70.15-73.43) \\
\hline & I change & $20.2 \%$ & $(|8.78-2| .7 \mid)$ \\
\hline & $\geq 2$ changes & $8.0 \%$ & $(7.02-9.01)$ \\
\hline \multirow[t]{2}{*}{ Age (years) } & $\begin{array}{l}\text { Mean } \\
(95 \%-\mathrm{Cl})\end{array}$ & 43.36 & $(42.96-43.76)$ \\
\hline & Median (IQR) & 44.00 & $(35.00-51.00)$ \\
\hline \multirow[t]{2}{*}{ EDSS } & $\begin{array}{l}\text { Mean } \\
(95 \%-\mathrm{Cl})\end{array}$ & 2.26 & $(2.21-2.32)$ \\
\hline & Median (IQR) & 2.00 & $(1.00-3.00)$ \\
\hline \multirow[t]{2}{*}{ Disease duration } & $\begin{array}{l}\text { Mean } \\
(95 \%-\mathrm{Cl})\end{array}$ & 8.78 & $(8.54-9.02)$ \\
\hline & Median (IQR) & 7.00 & $(3.00-12.00)$ \\
\hline \multirow[t]{2}{*}{ TSQM effectiveness } & $\begin{array}{l}\text { Mean } \\
(95 \%-\mathrm{Cl})\end{array}$ & 66.78 & $(65.92-67.65)$ \\
\hline & Median (IQR) & 66.67 & $(50.00-83.33)$ \\
\hline \multirow[t]{2}{*}{ TSQM side effects } & $\begin{array}{l}\text { Mean } \\
(95 \%-\mathrm{Cl})\end{array}$ & 74.57 & (73.55-75.58) \\
\hline & Median (IQR) & 81.25 & $(56.25-100)$ \\
\hline \multirow[t]{2}{*}{ TSQM convenience } & $\begin{array}{l}\text { Mean } \\
(95 \%-\mathrm{Cl})\end{array}$ & 70.24 & $(69.47-71.01)$ \\
\hline & Median (IQR) & 66.67 & $(55.56-88.89)$ \\
\hline
\end{tabular}

Notes: Disease-related and sociodemographic variables describing the patient population.

Abbreviations: $\mathrm{Cl}$, confidence interval; IQR, interquartile range (25th to 75th percentile); RRMS, relapsing-remitting multiple sclerosis; CIS, clinically isolated syndrome; DMT, disease-modifying treatment; IFN, interferon; GA, glatiramer acetate; EDSS, Expanded Disability Status Scale; TSQM, Treatment Satisfaction Questionnaire for Medication.
Compared to patients under current (IFN/GA) basic therapy, the 250 currently untreated patients presented increased disabilities (median EDSS 3 vs 2, p<0.001), more often relapses $(52.8 \%$ vs $37.5 \%, \mathrm{p}<0.001)$, higher fatigue $(60.3 \%$ vs $44.2 \%, \mathrm{p}<0.001)$ and depression rates $(22.5 \%$ vs $16.4 \%, \mathrm{p}=0.014)$ and longer disease durations (median 11 vs 7 years, $\mathrm{p}<0.001$ ). As the main reason for discontinuing treatment, 119 out of 250 patients (47.6\%) reported suffering from side effects, followed by lack of perceived effectiveness (17.2\%) and a general wish for a therapy break $(15.6 \%)$.

\section{Quality of Life and Treatment Satisfaction}

In total, the SF-36 mental component summary score was $42.87 \pm 12.12$. Mean SF score was $73.25 \pm 26.15$, RE was $68.28 \pm 28.96$, MH was $65.66 \pm 19.65$ and VT with 47.00 \pm 20.26 lowest. The physical component summary score was $45.49 \pm 12.03$ points. PF was $71.88 \pm 27.62$, followed by BP (70.47 \pm 27.94$), \mathrm{RF}(62.53 \pm 28.55)$ and GH (55.05 $\pm 21.41)$. An overview of all HRQoL scores stratified by the five treatment subgroups can be found in Table 2 . Corresponding Kruskal-Wallis tests (PF, RF, BP, GH, $\mathrm{VF}, \mathrm{SF}, \mathrm{RE}, \mathrm{MH}$ ) and ANOVAs (PCS, MCS) revealed statistically significant differences in means across DMT subgroups (Table 2). Corresponding effect sizes were medium (eta square of 0.012 for MCS) to almost large (eta square of 0.015 for PCS) and driven by the low HRQoL scores of currently untreated patients. In particular, currently untreated patients reported lowest HRQoL scores in every HRQoL domain as compared to the IFN/ GA group as a whole ( $<<0.01$, Table 2$)$. Within the IFN/ GA group, lowest HRQoL scores were observed for GA, while IFN treatment groups in general scored higher. However, corresponding effect sizes were low (eta squared for both PCS and MCS <0.006) and differences in HRQoL scores within the IFN/GA group predominantly non-significant.

HRQoL in our study was lower than in the general population: in every HRQoL dimension and treatment group, there was a statistically significant difference in mean SF-36 scores compared to subjects from a representative German sample ${ }^{32}$ (all corresponding $\mathrm{BCa}$ bootstrap intervals did not contain zero; data not shown).

Patient-reported satisfaction with treatment as measured by the TSQM was $74.57 \pm 27.73$ for side effects, $70.24 \pm 21.11$ for convenience and $66.78 \pm 23.54$ for effectiveness (Table 1). The general treatment satisfaction score 
Table 2 Health-Related Quality of Life (SF-36) Score Distributions in Patients on First-Line Treatment (N=2990)

\begin{tabular}{|c|c|c|c|c|c|c|}
\hline & & \multicolumn{5}{|l|}{ Current Medication } \\
\hline & & IFN beta-Ia i.m. (N=704) & IFN beta-I a s.c. $(\mathrm{N}=687)$ & IFN beta-Ib s.c. $(N=564)$ & GA $(\mathbf{N}=785)$ & No DMT $(\mathrm{N}=250)$ \\
\hline \multirow[t]{2}{*}{ PCS* } & Mean $(\mathrm{Cl})$ & $47.20(46.35-48.06)$ & 45.88 (44.97-46.78) & $45.32(44.27-46.37)$ & $44.99(44.12-45.87)$ & $41.40(39.76-43.04)$ \\
\hline & Median (IQR) & $48.54(39.66-56.61)$ & $47.85(37.54-56.07)$ & $46.92(36.14-55.95)$ & $45.88(36.05-55.40)$ & $41.78(30.96-51.24)$ \\
\hline \multirow[t]{2}{*}{ MCS* } & Mean $(\mathrm{Cl})$ & 44.35 (43.49-45.22) & $42.99(42.08-43.90)$ & $43.77(42.74-44.80)$ & $41.67(40.77-42.58)$ & $39.98(38.28-41.68)$ \\
\hline & Median (IQR) & $46.63(35.02-53.77)$ & $44.73(34.86-53.02)$ & $44.69(35.48-54.35)$ & $42.90(32.76-52.20)$ & $40.48(29.17-51.65)$ \\
\hline \multirow[t]{2}{*}{$\mathrm{PF} * *$} & Mean $(\mathrm{Cl})$ & 75.81 (73.90-77.72) & $73.02(70.99-75.05)$ & $70.35(67.95-72.75)$ & 71.46 (69.54-73.39) & $62.35(58.59-66.11)$ \\
\hline & Median (IQR) & $85.00(60.00-95.00)$ & $80.00(55.00-95.00)$ & $80.00(45.00-95.00)$ & $80.00(50.00-95.00)$ & $65.00(37.50-90.00)$ \\
\hline \multirow[t]{2}{*}{$\mathrm{RF}^{* *}$} & Mean $(\mathrm{Cl})$ & $66.87(64.81-68.92)$ & $64.69(62.58-66.81)$ & $62.98(60.58-65.37)$ & $60.06(58.04-62.08)$ & $50.91(47.38-54.43)$ \\
\hline & Median (IQR) & 68.75 (43.75-93.75) & $68.75(43.75-87.50)$ & $62.50(43.75-87.50)$ & $62.50(37.50-87.50)$ & $50.00(31.25-75.00)$ \\
\hline \multirow[t]{2}{*}{$\mathrm{BP} * *$} & Mean $(\mathrm{Cl})$ & $72.19(70.22-74.15)$ & $71.07(68.97-73.16)$ & 72.21 (69.9I-74.5I) & $69.28(67.26-71.30)$ & $63.82(59.78-67.68)$ \\
\hline & Median (IQR) & $74.00(51.00-100.0)$ & $74.00(51.00-100.0)$ & $74.00(51.00-100.0)$ & $72.00(41.00-100.0)$ & $62.00(41.00-100.0)$ \\
\hline \multirow[t]{2}{*}{$\mathrm{GH}^{* *}$} & Mean $(\mathrm{Cl})$ & $57.95(56.39-59.50)$ & $54.17(52.53-55.81)$ & 55.79 (53.98-57.59) & $54.23(52.73-55.73)$ & $50.20(47.45-52.95)$ \\
\hline & Median (IQR) & $57.00(42.00-72.00)$ & $52.00(37.00-72.00)$ & $57.00(40.00-72.00)$ & $52.00(37.00-72.00)$ & $47.00(35.00-67.00)$ \\
\hline \multirow[t]{2}{*}{$\mathrm{VT} \mathrm{T}^{* *}$} & Mean $(\mathrm{Cl})$ & $49.76(48.30-51.23)$ & $47.00(45.49-48.5 I)$ & $48.37(46.72-50.03)$ & $45.40(43.93-46.87)$ & $41.18(38.63-43.72)$ \\
\hline & Median (IQR) & $50.00(37.50-62.50)$ & $50.00(31.25-62.50)$ & $50.00(31.25-62.50)$ & $43.75(31.25-62.50)$ & $43.75(25.00-56.25)$ \\
\hline \multirow[t]{2}{*}{$\mathrm{SF}^{* * *}$} & Mean $(\mathrm{Cl})$ & $75.94(74.16-77.73)$ & 74.73 (72.79-76.76) & 74.62 (72.45-76.79) & $71.14(69.23-73.05)$ & $65.12(61.63-68.61)$ \\
\hline & Median (IQR) & $75.00(62.50-100.0)$ & $75.00(62.50-100.0)$ & $75.00(62.50-100.0)$ & $75.00(50.00-100.0)$ & $62.50(50.00-87.50)$ \\
\hline \multirow[t]{2}{*}{$\mathrm{RE}^{* *}$} & Mean $(\mathrm{Cl})$ & $72.36(70.27-74.46)$ & $68.74(66.61-70.87)$ & 69.34 (66.98-7I.7I) & $66.17(64.08-68.25)$ & $59.64(55.75-63.52)$ \\
\hline & Median (IQR) & $75.00(50.00-100.0)$ & $75.00(50.0-100.0)$ & $75.00(50.00-100.0)$ & $66.67(50.00-100.0)$ & 58.33 (33.33-91.67) \\
\hline \multirow[t]{2}{*}{$\mathrm{MH}^{* *}$} & Mean $(\mathrm{Cl})$ & $67.33(65.90-68.76)$ & $65.50(64.07-66.93)$ & $67.16(65.56-68.77)$ & $64.47(63.05-65.88)$ & $61.80(59.20-64.40)$ \\
\hline & Median (IQR) & $70.00(55.00-83.33)$ & $70.00(50.00-80.00)$ & $70.00(55.00-85.00)$ & $65.00(50.00-80.00)$ & $65.00(45.00-80.00)$ \\
\hline \multirow[t]{2}{*}{ TSQM global* } & Mean $(\mathrm{Cl})$ & 72.35 (70.82-73.87) & $72.3 \mathrm{I}(70.8 \mathrm{I}-73.82)$ & 72.63 (70.87-74.39) & $71.45(69.99-72.91)$ & $45.75(4 \mid .25-50.25)$ \\
\hline & Median (IQR) & 78.57 (57.14-85.7I) & $78.57(57.14-85.7 I)$ & 78.57 (57.14-92.86) & $71.43(57.14-85.71)$ & $42.86(2|.43-7| .43)$ \\
\hline
\end{tabular}

Notes: *ANOVA result: $p<0.0$ I (IFN beta-la i.m. vs IFN beta-la s.c. vs IFN beta-lb s.c. vs GA vs No DMT), two sample $t$-test result: $p<0.0$ I (No DMT vs IFN/GA). **Kruskal-Wallis result: $\mathrm{p}<0.0$ I (IFN beta-la i.m. vs IFN beta-la s.c. vs IFN beta-lb s.c. vs GA vs No DMT), Mann-Whitney U-test result: $p<0.0$ I (No DMT vs IFN/GA). Abbreviations: $\mathrm{Cl}$, (95\%) confidence interval; IQR, interquartile range (25th to 75th percentile); DMT, disease-modifying treatment; IFN, interferon; GA, glatiramer acetate; i.m., intramuscular; s.c., subcutaneous; PCS, Physical Component Summary Score; MCS, Mental Component Summary Score; PF, physical functioning; RF, role-physical; BP, bodily pain; GH, general health; VT, vitality; SF, social functioning; RE, role-emotional; MH, mental health; TSQM, Treatment Satisfaction Questionnaire for Medication.

was $70.42 \pm 22.20$. While treatment satisfaction did not differ within the IFN/GA group, considerable lower treatment satisfaction scores were observed for currently untreated patients (Table 2 for TSQM global).

\section{Bivariate Associations}

Rank correlations of the SF-36 and TSQM were consistently positive and ranged from relatively low to medium effect sizes (Table 3).

Satisfaction with medication in terms of effectiveness correlated more strongly with all of the HRQoL dimensions than either convenience, side effects or global satisfaction.
The corresponding range was $r=0.272$ to $r=0.373$ for the eight single scales and $\mathrm{r}=0.310$ to $\mathrm{r}=0.352$ for the summary measures. The strongest association was observed for $\mathrm{GH}$ and TSQM effectiveness ( $r=0.373$, Table 3 ). TSQM convenience was lower correlated to every HRQoL dimension than all the other TSQM domains (ranging from $\mathrm{r}=0.052$ to $\mathrm{r}=0.227)$.

To account for potential factors affecting the HRQoL scores while quantifying the influence of TSQM effectiveness, side effects and convenience, multivariate analysis techniques were performed for both PCS and MCS as representatives of HRQoL. All 16 variables listed in 
Table 3 Rank Correlation Results for Treatment Satisfaction and Health-Related Quality of Life (N=2990)

\begin{tabular}{|l|l|l|l|l|l|l|l|l|l|l|}
\hline & PCS & MCS & PF & RF & BP & GH & VT & SF & RE & MH \\
\hline TSQM effectiveness & 0.352 & 0.310 & 0.283 & 0.338 & 0.272 & 0.373 & 0.326 & 0.321 & 0.274 & 0.286 \\
TSQM side effects & 0.199 & 0.247 & 0.121 & 0.181 & 0.259 & 0.208 & 0.255 & 0.231 & 0.193 & 0.219 \\
TSQM convenience & 0.102 & 0.227 & 0.052 & 0.103 & 0.121 & 0.189 & 0.189 & 0.181 & 0.162 & 0.215 \\
TSQM global satisfaction & 0.270 & 0.314 & 0.199 & 0.251 & 0.232 & 0.334 & 0.295 & 0.294 & 0.250 & 0.300 \\
\hline
\end{tabular}

Abbreviations: TSQM, Treatment Satisfaction Questionnaire for Medication; PCS, Physical Component Summary Score; MCS, Mental Component Summary Score; PF, physical functioning; RF, role-physical; BP, bodily pain; GH, general health; VT, vitality; SF, social functioning; RE, role-emotional; $M H$, mental health.

Table 1 were included in one approach in classification and regression trees (Figures 1 and 2; see Appendix 1 for PF, RF, BP, GH, VT, SF, RE and MH). Next, the optimal subset of explanatory variables based on the LASSO (Appendix 2) was included in a linear model (Table 4).

\section{Cross-Sectional Predictors for PCS}

Higher EDSS scores were highly influential on decreased PCS scores according to the regression tree analysis (Figure 1, layer 1). Further, being employed, higher TSQM effectiveness and side effect scores, absence of fatigue and decreasing age were positively associated with PCS (all p<0.001).

Eight selected explanatory variables derived from the LASSO (Appendix 2) resulted in a multivariate regression formula holding over the entire data space. The crosssectional predictor with the highest explanatory power was disability as measured by the EDSS (standardized beta $(b)=-0.408)$. Next, employment status $(b=+0.163)$, age $(b=-0.159)$ as well as TSQM side effect $(b=+0.146)$ and TSQM effectiveness $(b=+0.137)$ followed (Table 4$)$.

\section{Cross-Sectional Predictors for MCS}

For mental health, depression was a main determinant (Figure 2, layer 1). The absence of a major depressive episode, apparent in a sixth of the patients, was significantly associated with higher MCS scores. Furthermore, better treatment satisfaction in terms of effectiveness, side effects and convenience, absence of fatigue and being in work were positively associated with mental health (all $\mathrm{p}<0.001$; Figure 2).

The LASSO selected nine explanatory variables for best cross-sectional prediction of the mental HRQoL dimension on a global level (Appendix 2): TSQM effectiveness, TSQM side effects, TSQM convenience, being out of work, fatigue, depression, EDSS, presence of relapses and DMT-group. All these variables were significantly related to decreased MCS on a $\mathrm{p}<0.001$ level, except EDSS $(\mathrm{p}<0.05)$ and treatment group ( $p>0.05$; Table 4 ). Depression had the strongest impact on reduced MCS scores $(b=-0.234)$, followed by TSQM side effects $(b=+0.152)$, TSQM effectiveness $(b=+0.131)$, fatigue $(b=-0.130)$ and TSQM convenience $(b=+0.116)$.

Results from global regression models (Table 4) were largely consistent with the conclusion drawn from tree analyses based on recursive partitioning (Figures 1 and 2, Appendix 1). The relative impact of gender, disease course (RRMS versus CIS), disease duration and number of treatment changes were limited in both MCS and PCS multivariate models (Appendix 2).

Stratified analyses by DMT-group indicated that the selected multivariate models and their determinants were robust across treatment subgroups (regression models not shown).

Results from tree analysis did not give concern for major interaction effects to be taken into account at the global level. As the cross-sectional predictors for regression modelling were chosen by the LASSO, overfitting was minimized and collinearity not considered as an issue in the multivariate models (all variance inflation factors (VIF) <1.5). The PCS models explained more than half of the variance, whilst the MCS models could only account for around a quarter of the variation (Table 4).

\section{Discussion}

In this large cross-sectional study, we described HRQoL in almost three thousand patients with RRMS receiving injectable IFN or GA and demonstrated that there is a considerable positive association to self-reported treatment satisfaction. Furthermore, adjusted for other (well known) determinants of HRQoL, the independent crosssectional predictive value of treatment satisfaction in terms of side effects and effectiveness could be quantified for both the physical and mental health dimensions of wellbeing through a data-driven approach.

Although HRQoL and treatment satisfaction are interrelated, both PROs remain independent classes of constructs. ${ }^{5}$ 


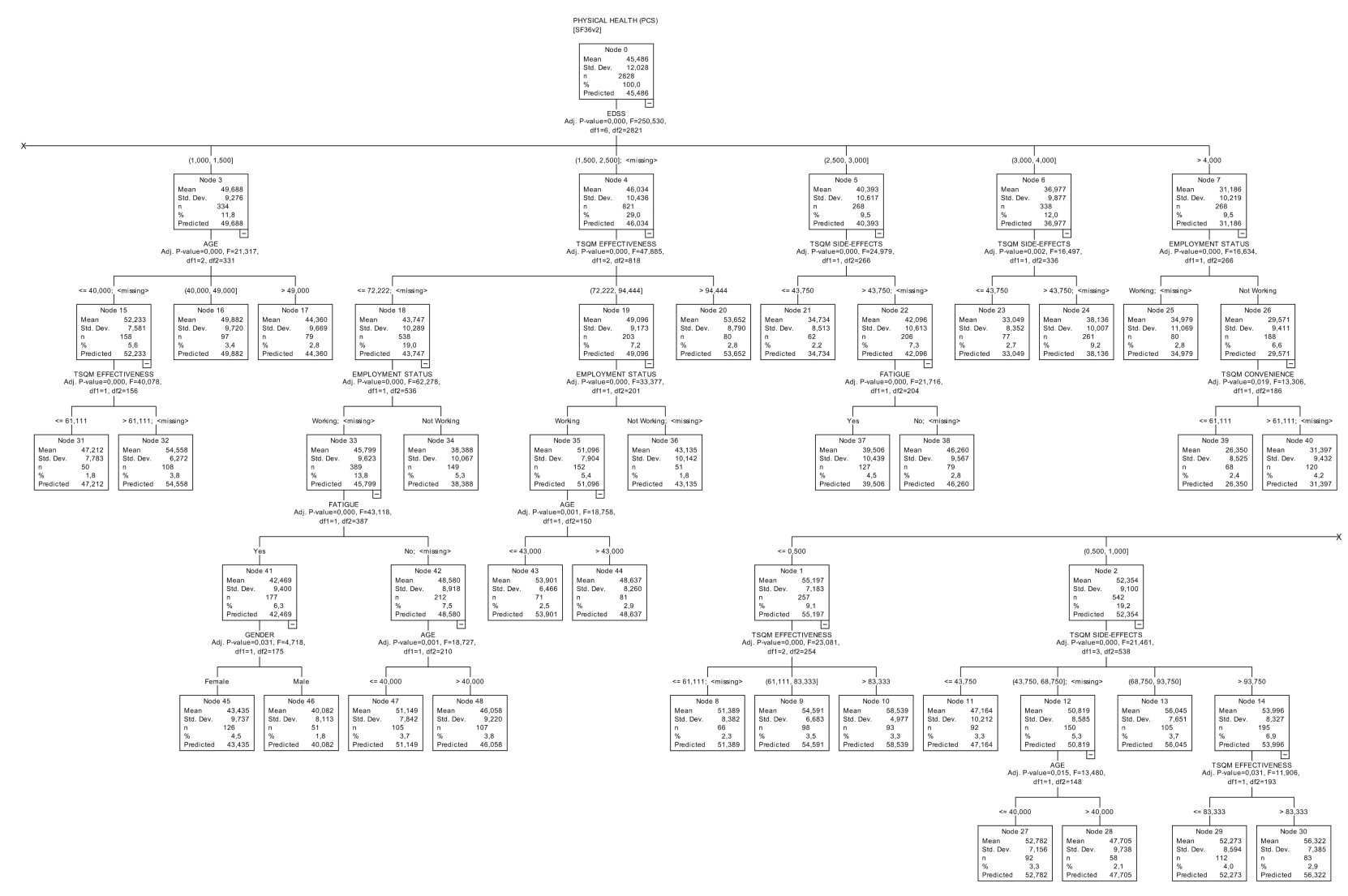

Figure I Regression tree for the Physical Component Summary (PCS).

Notes: The tree was recursively grown based on the restriction of a minimum node size of 50 and a maximum number of 5 tree layers. Variables included: TSQM effectiveness, TSQM side effects, TSQM convenience, type of medication (DMT), number of medication changes, duration of medication intake, disease duration, disease course (CIS, RRMS), EDSS, relapses in the last 12 months, fatigue, depression, gender, age, currently living with a partner, employment status.

Mild and moderate effect sizes (correlations) found in our study demonstrate this distinct character. HRQoL as state of one's health is more universal and independent of particular life events and circumstances, whilst treatment satisfaction comprises judgement about more specific set of treatmentrelated experiences and events. ${ }^{33}$ From a practical point of view, HRQoL can be measured prior to starting a medication, whilst treatment satisfaction cannot be assessed at this stage.

Concerning the generic SF-36 instrument, our study reported significantly lower HRQoL scores than reference values extracted from the German population. A result that has been shown in comparable studies in different regions before. ${ }^{3,34,35}$ HRQoL was especially low in the group of patients who were currently no longer on first-line treatment. Accordingly, the same subpopulation not only showed decreased HRQoL but was also least satisfied with the overall treatment and in regard to convenience, side effects and effectiveness. This is obviously in line with the evidence that satisfaction is related to adherence and motivation to adhere to DMT is further connected to HRQoL. ${ }^{14,36-38}$

\section{Association of HRQoL and Treatment Satisfaction in MS}

To the best of our knowledge, no study demonstrated the association of various HRQoL and treatment satisfaction dimensions in an MS setting comparable to ours before. As a secondary objective, Mekies and colleagues presented bivariate (unadjusted) associations using only the global treatment satisfaction domain as a dichotomized outcome (TSQM global $\geq 75$ and $<75$ ) and the EuroQoL questionnaire on the other hand. ${ }^{10}$ In contrast, we were able to present a fourteenfold higher sample size and data-driven multivariate analyses based on three distinct TSQM dimensions each covering the full scaling range while adjusting for other well-known risk factors potentially affecting HRQoL. However, applying a dichotomous 75-points-cutoff for global satisfaction to our data, we were able to reflect the authors' results using the 


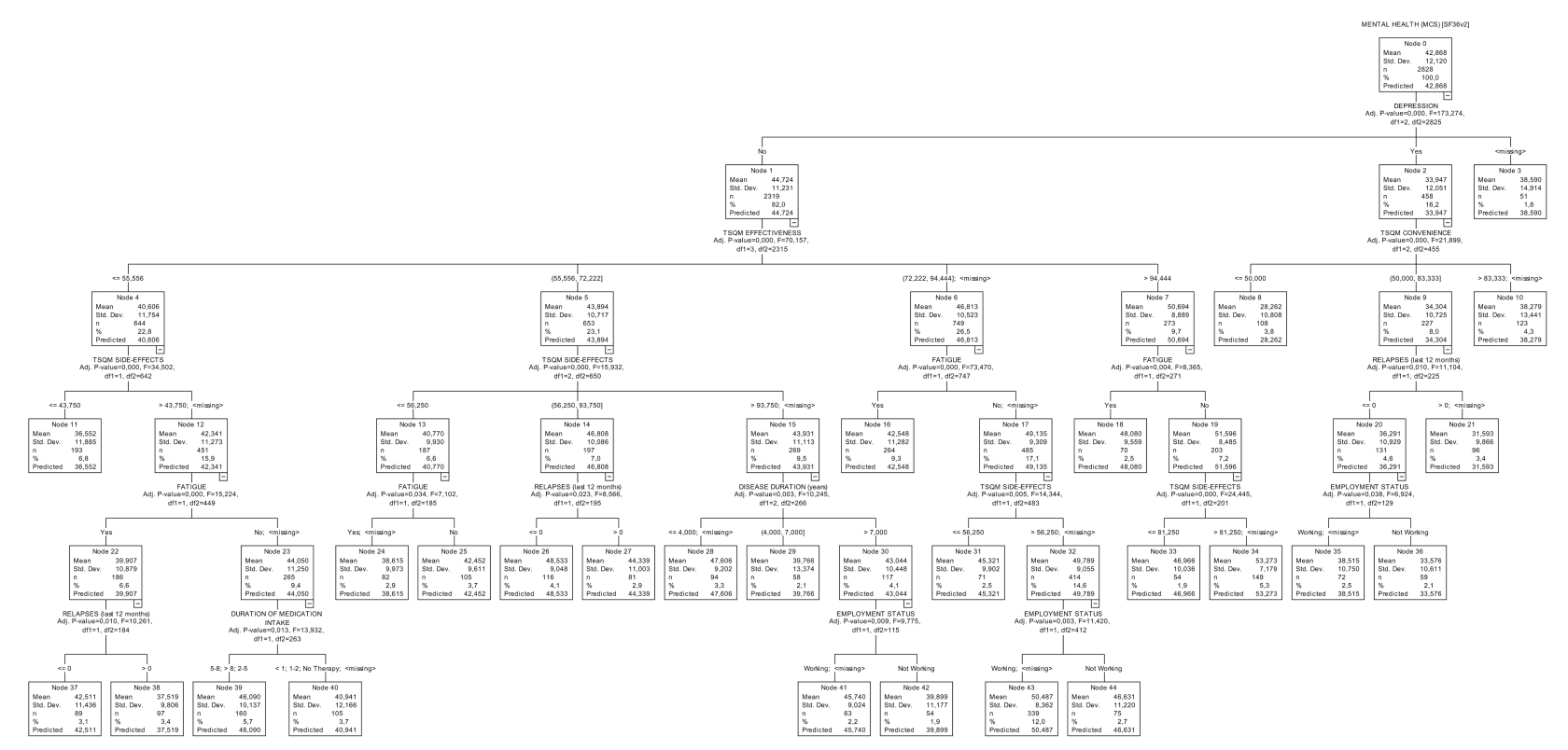

Figure 2 Regression tree for the Mental Component Summary (MCS).

Notes: The tree was recursively grown based on the restriction of a minimum node size of 50 and a maximum number of 5 tree layers. Variables included: TSQM effectiveness, TSQM side effects, TSQM convenience, type of medication (DMT), number of medication changes, duration of medication intake, disease duration, disease course (CIS, RRMS), EDSS, relapses in the last 12 months, fatigue, depression, gender, age, currently living with a partner, employment status.

SF-36 health dimensions. As to be expected, the mean HRQoL scores were significantly higher in the more satisfied group (TSQM global score $\geq 75$ ) in all eight health domains of the SF36 compared to those patients unsatisfied (TSQM global score $<75$ ) with treatment (all $\mathrm{p}<0.001$; data not shown). The summary aggregates PCS and MCS were also significantly higher in patients who were generally more satisfied with their therapy compared to their unsatisfied counterparts (48.48 \pm 11.46 vs $42.87 \pm 11.85$ for PCS and $46.16 \pm 11.26$ vs 39.99 \pm 11.97 for MCS; both $\mathrm{p}<0.001$ ). Nevertheless, it must be noted that a dichotomized consideration of the TSQM outcomes is not an advisable approach to operationalize treatment satisfaction since a threshold for low and high treatment satisfaction has never been validated. Likewise, there is no reason to assume that such a dichotomization at 75 should be equally applied to all TSQM subscores.

Other authors assessed treatment satisfaction using the TSQM in tandem with HRQoL measures for MS patients under treatment but did not examine the association of these measures due to deviating study objectives. ${ }^{13,36}$

\section{Association of HRQoL and Treatment Satisfaction in Other Medical Fields}

Nonetheless, the association of HRQoL and treatment satisfaction has been discussed and shown in other medical fields before. ${ }^{33,39-43}$
Firstly, the correlation analysis of our study revealed that satisfaction was strongest associated with the SF-36 domain of GH. This corresponds with the findings presented by Nicolucci and colleagues, who evaluated correlates of treatment satisfaction and quality of life in a population of two and a half thousand patients diagnosed with type 2 diabetes. ${ }^{39}$

In a study on gout patients published by Khanna et al, it was rather the opposite observed: GH was considerable lower correlated to satisfaction as compared to other domains. ${ }^{40}$ One factor that might contribute to an explanation is that patients suffering from MS are known to have lower HRQoL than patients from many other (chronic) diseases. ${ }^{44}$ Nevertheless, as in our study, the same authors demonstrated that the mental domain (MCS) showed higher bivariate associations to TSQM side effects, TSQM convenience and TSQM global satisfaction than the physical domain (PCS). Also in line with our findings, PCS was slightly higher correlated to the TSQM effectiveness domain than MCS.

Congruent to our results, Al-Jabi and colleagues found in a study on over four hundred hypertensive patients not only positive correlations between TSQM domains and HRQoL $(p<0.001)$ but also identified general treatment satisfaction as a main independent predictor after adjustment for covariates other than MS treatment satisfaction $(\mathrm{p}<0.001){ }^{41}$ 
Table 4 Cross-Sectional Predictors of Physical Health and Mental Health Domains: Multivariate Regression Analyses $(\mathrm{N}=2990)$

\begin{tabular}{|c|c|c|c|c|}
\hline & \multicolumn{2}{|c|}{$\begin{array}{l}\text { PCS Model } \\
(\mathrm{N}=2439)\end{array}$} & \multicolumn{2}{|c|}{$\begin{array}{l}\text { MCS Model } \\
(N=2439)\end{array}$} \\
\hline & b & P-value & b & P-value \\
\hline TSQM effectiveness & +0.137 & $<0.001$ & +0.131 & $<0.001$ \\
\hline TSQM side effects & +0.146 & $<0.001$ & +0.152 & $<0.001$ \\
\hline TSQM convenience & & & +0.116 & $<0.001$ \\
\hline Age & -0.159 & $<0.001$ & & \\
\hline Employment status ${ }^{\mathrm{a})}$ & +0.163 & $<0.001$ & +0.075 & $<0.001$ \\
\hline Fatigue ${ }^{\text {b) }}$ & -0.117 & $<0.001$ & -0.130 & $<0.001$ \\
\hline Depression ${ }^{\text {b) }}$ & -0.059 & $<0.001$ & -0.234 & $<0.001$ \\
\hline Disability & -0.408 & $<0.001$ & -0.052 & 0.012 \\
\hline Relapses ${ }^{c)}$ & -0.077 & $<0.001$ & -0.085 & $<0.001$ \\
\hline Current DMT d) & & & +0.030 & 0.103 \\
\hline$R / R^{2}$ adjusted & \multirow{2}{*}{\multicolumn{2}{|c|}{$0.726 / 0.526$}} & \multicolumn{2}{|c|}{$0.502 / 0.249$} \\
\hline Maximum VIF & & & \multicolumn{2}{|l|}{$<1.4$} \\
\hline
\end{tabular}

Notes: Standardized beta coefficients (b) and p-values of LASSO-selected crosssectional predictors in linear regression models with PCS and MCS as dependent variables. For a one standard deviation increase in a given variable, holding the other explanatory variables constant, the outcome variable (PCS, MCS) changes by the standardized coefficient number of standard deviations. a) Working vs not working, b) Yes vs no, c) More than one relapse vs no relapse in the past 12 months, d) IFN/GA vs No DMT. Abbreviations: b, standardized beta coefficients; TSQM, Treatment Satisfaction Questionnaire for Medication; DMT, disease-modifying treatment; IFN, interferon; GA, glatiramer acetate; PCS, Physical Component Summary Score; MCS, Menta Component Summary Score; VIF, variance inflation factor; R, coefficient of determination; LASSO, least absolute shrinkage and selection operator.

Delestras and colleagues investigated the relationship between satisfaction with medication and HRQoL in a population of 190 patients with various chronic diseases. ${ }^{42}$ In a multivariable regression model with each PCS and MCS as a dependent variable and treatment satisfaction domains as explanatory variables (measured by the Sat-Med-Q), effectiveness and side effects domains were strongest associated with HRQoL in both models (all $\mathrm{p}<0.01$ ). This is clearly in line with our findings. Using the TSQM Version II questionnaire instead of the Sat-Med-Q yielded in the result that TSQM convenience was now identified to be significantly related to MCS, but not to PCS. This is also consistent with our regression analyses. On the other hand, the TSQM effectiveness domain was now less influential as compared to our MS specific analyses.

\section{Explanatory Factors for HRQoL Other Than Treatment Satisfaction}

When analyzing explanatory factors other than treatment satisfaction that impact on HRQoL, disability as measured by the EDSS was the major determinant for PCS, which has been demonstrated in many MS studies using the SF36 before. $^{8}$ This was not only true in the regression models (Table 4) and tree analyses (Figure 1) of PCS but also observed for all single physical related domains PF, RF, BP and GH (Appendix 1). Age was further identified as major independent exploratory variable for impaired PCS but not for MCS, which has also been shown in patients with MS before. ${ }^{45}$ The known effects of fatigue and depression on decreased HRQoL in MS were also reflected in our population. ${ }^{46}$ Additionally, Nourbakhsh and colleagues found that both the severity of fatigue and depression were related to MCS, but for PCS, fatigue was more influential. ${ }^{9}$ It is interesting to note that our regression models were in line with this finding although we only used a binary depression and fatigue rating by the attending physician compared to the higher dimensional rating scales utilized by the authors.

Beyond fatigue and depression, Barin and colleagues identified gait problems, spasticity, balance problems or tremor as most relevant MS-symptoms (out of 20) affecting the HRQoL in people with MS. ${ }^{47}$ These measures of the disease burden were not collected in our study but might have been partly captured by the EDSS as measure of MS disability.

Factors such as duration of medication intake or disease duration were not part of the main explanatory variables associated with HRQoL. However, they have been shown to be significantly associated with treatment satisfaction. ${ }^{11}$ Consequently, in our sparse cross-sectional predicting models of HRQoL, TSQM variables might have overshadowed these factors.

Post-authorization studies altogether suggest that there is stabilizing (or increasing) impact of IFN and GA on HRQoL. ${ }^{48}$ In our study, there were differences in DMT-groups regarding the HRQoL outcomes, especially the No-DMT group scored lower. In multivariate analyses, the DMT-group variable did not show major impact. This was to be expected, as our study was neither designed to assess medication effects nor it was longitudinal by design. In addition, only $8.4 \%$ of patients were currently without treatment. However, patients in the No-DMT group presented significantly decreased treatment satisfaction and increased rates of disability, fatigue and depression. Consequently, these factors were part of the multivariate HRQoL models and have been identified as key explanatory variables for decreased HRQoL.

\section{Strengths and Limitations}

One limitation of the current study is the cross-sectional design. As a result, temporal and causal relationships cannot 
be established. Another limitation may arise from the documentation of self-reported satisfaction from the currently untreated group. Compared to patients who currently received treatment, they may be more prone to some recall bias due to their retrospective assessment of treatment satisfaction. The THEPA-MS survey further only focused on first-generation, classical injectable first-line treatments as opposed to newer generation DMTs, which in particular may be an option for some patients who did not benefit from IFN/GA. On the other hand, since participation was voluntary, it cannot be excluded that patients who showed above-average HRQoL or therapy satisfaction were more likely to participate in our study. Also, if attending physicians erroneously selected patients on the basis of their state of well-being or disease severity, there may be some distortion in patient selection. Mean EDSS was 2.26 (median 2) indicating some underrepresentation of more severe patients. As strength, the survey represented a real-life setting with patients from all parts of Germany. Consequently, currently untreated patients were not excluded, nor other exclusion criteria such as a minimum time on current treatment were introduced. In particular, the demographic characteristics of our study match with those of an expected MS-population, where women get the disorder three to four times more than men and patients are usually diagnosed between the ages of 20 and 40 years. Finally, with approximately 3000 patients our explorative analyses were well powered.

\section{Implications for Future Research}

Future research may further address to the direction and strength of association of treatment satisfaction and HRQoL over time. Longitudinal study designs may contribute here to a clarification of the directionality. Further insights in the association of treatment satisfaction and HRQoL could be given when focusing on newer generation (infusion or oral) DMTs or when replacing clinician-rated by patient-reported measures of disability, fatigue or depression. ${ }^{16}$

\section{Conclusion}

Overall, our research supports the aspiration of modern medicine to consider the importance of the patient perspective in clinical decision-making, as opposed to the more paternalistic approach which is based primarily on observer ratings by health professionals. We provided insights on the inter-relationship between HRQoL and MS treatment satisfaction alongside with other independent explanatory variables within a real-world setting. Treatment satisfaction in terms of perceived effectiveness, perceived side-effects and perceived convenience was part of the main determinants of HRQoL. This may offer enhancements to clinical practice in that they support an efficient and holistic approach relative to management of the MS disease. Besides the opportunity to affect fatigue, depression and disability as primary determinants of impaired HRQoL, emphasizing treatment satisfaction (and more broadly patient satisfaction and needs) may enhance HRQoL. Consequently, persistence and adherence to treatment may be mediated or moderated. For example, the introduction of regular collection and evaluation of treatment satisfaction measures in everyday clinical practice may serve as an additional early warning system for treatment success at risk.

\section{Funding}

The study was funded by Sanofi Genzyme, Germany. The funder had no role in the collection, analyses, or interpretation of data; in the writing of the manuscript, or in the decision to publish the results.

\section{Disclosure}

Rocco Haase has received speaker fee from Sanofi and travel grant from Teva. Jennifer S. Kullmann is an employee of Sanofi. Tjalf Ziemssen received personal compensation from Almirall, Biogen, Bayer, Celgene, Merck, Novartis, Roche, Sanofi and Teva for consulting services. Tjalf Ziemssen received additional financial support for research activities from Bayer, BAT, Biogen, Novartis, Teva, and Sanofi Aventis. The authors report no other conflicts of interest in this work.

\section{References}

1. Noseworthy JH, Lucchinetti C, Rodriguez M, Weinshenker BG. Multiple sclerosis. $N$ Engl $J$ Med. 2000;343(13):938-952. doi:10.1056/NEJM200009283431307

2. Dobson R, Giovannoni G. Multiple sclerosis - a review. Eur J Neurol. 2019;26(1):27-40. doi:10.1111/ene.13819

3. Benito-Leon J, Morales JM, Rivera-Navarro J, Mitchell A. A review about the impact of multiple sclerosis on health-related quality of life. Disabil Rehabil. 2003;25(23):1291-1303. doi:10.1080/096382803 10001608591

4. Karimi M, Brazier J. Health, health-related quality of life, and quality of life: what is the difference? PharmacoEconomics. 2016;34 (7):645-649. doi:10.1007/s40273-016-0389-9

5. Shikiar R, Rentz AM. Satisfaction with medication: an overview of conceptual, methodologic, and regulatory issues. Value Health. 2004;7 (2):204-215. doi:10.1111/j.1524-4733.2004.72252.x

6. Atkinson MJ, Kumar R, Cappelleri JC, Hass SL. Hierarchical construct validity of the treatment satisfaction questionnaire for medication (TSQM version II) among outpatient pharmacy consumers. Value Health. 2005;8(Suppl 1):S9-S24. doi:10.1111/j.1524-4733.2005. 00066.x 
7. Janardhan V, Bakshi R. Quality of life in patients with multiple sclerosis: the impact of fatigue and depression. J Neurol Sci. 2002;205(1):51-58. doi:10.1016/S0022-510X(02)00312-X

8. Berrigan LI, Fisk JD, Patten SB, et al. Health-related quality of life in multiple sclerosis: direct and indirect effects of comorbidity. Neurology. 2016;86(15):1417-1424. doi:10.1212/WNL.00000000 00002564

9. Nourbakhsh B, Julian L, Waubant E. Fatigue and depression predict quality of life in patients with early multiple sclerosis: a longitudinal study. Eur J Neurol. 2016;23(9):1482-1486. doi:10.1111/ene.13102

10. Mekies C, Heinzlef O, Jenny B, Ramelli AL, Clavelou P. Treatment satisfaction and quality of life in patients treated with fingolimod Patient Prefer Adherence. 2018;12:899-907. doi:10.2147/PPA. S144021

11. Haase R, Kullmann JS, Ziemssen T. Therapy satisfaction and adherence in patients with relapsing-remitting multiple sclerosis: the THEPA-MS survey. Ther Adv Neurol Disord. 2016;9(4):250-263. doi: $10.1177 / 1756285616634247$

12. Barbosa CD, Balp MM, Kulich K, Germain N, Rofail D. A literature review to explore the link between treatment satisfaction and adherence, compliance, and persistence. Patient Prefer Adherence. 2012;6:39-48. doi:10.2147/PPA.S24752

13. Saiz A, Mora S, Blanco J. Treatment compliance with first line disease-modifying therapies in patients with multiple sclerosis Compliance study. Neurol (English Edition). 2015;30(4):214-222. doi:10.1016/j.nrleng.2015.03.003

14. Grytten N, Aarseth JH, Espeset K, et al. Health-related quality of life and disease-modifying treatment behaviour in relapsing-remitting multiple sclerosis-a multicentre cohort study. Acta Neurol Scand Suppl. 2012;126(195):51-57. doi:10.1111/ane. 12033

15. Steinberg SC, Faris RJ, Chang CF, Chan A, Tankersley MA. Impact of adherence to interferons in the treatment of multiple sclerosis: a non-experimental, retrospective, cohort study. Clin Drug Investig. 2010;30(2):89-100. doi:10.2165/11533330-0000 00000-00000

16. Khurana V, Sharma H, Afroz N, Callan A, Medin J. Patient-reported outcomes in multiple sclerosis: a systematic comparison of available measures. Eur J Neurol. 2017;24(9):1099-1107. doi:10.1111/ ene. 13339

17. Ting J, Liu Y, Petrillo J, Giannattasio G, Sabatella G. Treatment satisfaction with disease modifying therapies in multiple sclerosis: a systematic review of studies using the treatment satisfaction questionnaire for medication (Tsqm). Value Health. 2015;18(7):A760 A761. doi:10.1016/j.jval.2015.09.2484

18. Polman $\mathrm{CH}$, Reingold SC, Edan G, et al. Diagnostic criteria for multiple sclerosis: 2005 revisions to the "McDonald criteria". Ann Neurol. 2005;58(6):840-846. doi:10.1002/ana.20703

19. DGN/KKNMS. German Neurological Society (DGN). Leitlinie zur Diagnose und Therapie der MS [Guidelines for MS Diagnosis and Treatment]. 2014.

20. Kurtzke JF. Rating neurologic impairment in multiple sclerosis: an expanded disability status scale (EDSS). Neurology. 1983;33 (11):1444-1452. doi:10.1212/WNL.33.11.1444

21. Ware JE, Kosinski M, Bjorner JB, Turner-Bowker DM, Gandek B, Maruish ME. User's Manual for the SF-36v2 Health Survey. 2nd ed. Quality Metric Lincoln (RI); 2007.

22. Morfeld M, Bullinger M, Nantke J, Brahler E. Die version 2.0 des SF-36 health survey: ergebnisse einer bevölkerungsrepräsentativen Studie [The version 2.0 of the SF-36 Health Survey: results of a population-representative study]. Soz Praventivmed. 2005;50 (5):292-300. doi:10.1007/s00038-005-4090-6

23. Atkinson MJ, Sinha A, Hass SL, et al. Validation of a general measure of treatment satisfaction, the Treatment Satisfaction Questionnaire for Medication (TSQM), using a national panel study of chronic disease. Health Qual Life Outcomes. 2004;2(1):12. doi:10.1186/1477-7525-2-12
24. Vermersch P, Hobart J, Dive-Pouletty C, Bozzi S, Hass S, Coyle PK. Measuring treatment satisfaction in MS: is the treatment satisfaction questionnaire for medication fit for purpose? Mult Scler (Houndmills, Basingstoke, England). 2017;23(4):604-613. doi:10.1177/13524585 16657441

25. Cohen J. Statistical Power Analysis for the Behavioral Sciences. 2nd edn. Hillsdale, NJ: Lawrence Erlbaum Associates; 1988.

26. Walters SJ, Campbell MJ. The use of bootstrap methods for analysing health-related quality of life outcomes (particularly the SF-36). Health Qual Life Outcomes. 2004;2(1):70. doi:10.1186/1477-75252-70

27. Gignac GE, Szodorai ET. Effect size guidelines for individual differences researchers. Pers Individ Dif. 2016;102:74-78. doi:10.1016/j. paid.2016.06.069

28. Biggs D, De Ville B, Suen E. A method of choosing multiway partitions for classification and decision trees. J Appl Stat. 1991;18 (1):49-62. doi:10.1080/02664769100000005

29. Meulman J, Heiser W. IBM SPSS categories 21; 2012. Available from: ftp://public.dhe.ibm.com/software/analytics/spss/documenta tion/statistics/21.0/en/client/Manuals/IBM_SPSS_Categories.pdf.

30. Tibshirani R. Regression shrinkage and selection via the lasso. $J$ R Stat Soc B Methodol. 1996;58(1):267-288. doi:10.1111/j.25176161.1996.tb02080.x

31. Walter S, Tiemeier H. Variable selection: current practice in epidemiological studies. Eur J Epidemiol. 2009;24(12):733-736. doi:10.1007/s10654-009-9411-2

32. Ellert U, Kurth BM. Health related quality of life in adults in Germany: results of the German health interview and examination survey for adults (DEGS1). Bundesgesundheitsblatt Gesundheitsforschung Gesundheitsschutz. 2013;56(5-6):643-649. doi:10.1007/s00103-013-1700-y

33. Asadi-Lari M, Tamburini M, Gray D. Patients' needs, satisfaction, and health related quality of life: towards a comprehensive model. Health Qual Life Outcomes. 2004;2(1):32. doi:10.1186/1477-7525-2-32

34. Amtmann D, Bamer AM, Kim J, Chung H, Salem R. People with multiple sclerosis report significantly worse symptoms and health related quality of life than the US general population as measured by PROMIS and NeuroQoL outcome measures. Disabil Health J. 2018;11(1):99-107. doi:10.1016/j.dhjo.2017.04.008

35. Nortvedt MW, Riise T, Myhr KM, Nyland HI. Quality of life in multiple sclerosis: measuring the disease effects more broadly. Neurology. 1999;53(5):1098-1103. doi:10.1212/WNL.53.5.1098

36. Coyle PK, Khatri B, Edwards KR, et al. Patient-reported outcomes in relapsing forms of MS: real-world, global treatment experience with teriflunomide from the Teri-PRO study. Mult Scler Relat Disord. 2017;17:107-115. doi:10.1016/j.msard.2017.07.006

37. Treadaway K, Cutter G, Salter A, et al. Factors that influence adherence with disease-modifying therapy in MS. J Neurol. 2009;256 (4):568-576. doi:10.1007/s00415-009-0096-y

38. Twork S, Nippert I, Scherer P, Haas J, Pohlau D, Kugler J. Immunomodulating drugs in multiple sclerosis: compliance, satisfaction and adverse effects evaluation in a German multiple sclerosis population. Curr Med Res Opin. 2007;23(6):1209-1215. doi:10.1185/ $030079907 \mathrm{X} 188125$

39. Nicolucci A, Cucinotta D, Squatrito S, et al. Clinical and socio-economic correlates of quality of life and treatment satisfaction in patients with type 2 diabetes. Nutr Metab Cardiovasc Dis. 2009;19 (1):45-53. doi:10.1016/j.numecd.2007.12.005

40. Khanna PP, Shiozawa A, Walker V, et al. Health-related quality of life and treatment satisfaction in patients with gout: results from a cross-sectional study in a managed care setting. Patient Prefer Adherence. 2015;9:971-981. doi:10.2147/PPA.S83700

41. Al-Jabi SW, Zyoud SH, Sweileh WM, et al. Relationship of treatment satisfaction to health-related quality of life: findings from a cross-sectional survey among hypertensive patients in Palestine. Health Expect. 2015;18(6):3336-3348. doi:10.1111/hex.12324 
42. Delestras S, Roustit M, Bedouch P, et al. Comparison between two generic questionnaires to assess satisfaction with medication in chronic diseases. PLoS One. 2013;8(2):e56247. doi:10.1371/journal. pone.0056247

43. Chen H, Rosenzweig EB, Gotzkowsky SK, Arneson C, Nelsen AC, Bourge RC. Treatment satisfaction is associated with improved quality of life in patients treated with inhaled treprostinil for pulmonary arterial hypertension. Health Qual Life Outcomes. 2013;11(1):31. doi:10.1186/1477-7525-11-31

44. Hermann BP, Vickrey B, Hays RD, et al. A comparison of health-related quality of life in patients with epilepsy, diabetes and multiple sclerosis. Epilepsy Res. 1996;25(2):113-118. doi:10.1016/ 0920-1211(96)00024-1

45. Rezapour A, Almasian Kia A, Goodarzi S, Hasoumi M, Nouraei Motlagh S, Vahedi S. The impact of disease characteristics on multiple sclerosis patients' quality of life. Epidemiol Health. 2017;39: e2017008. doi:10.4178/epih.e2017008
46. Ziemssen T. Multiple sclerosis beyond EDSS: depression and fatigue. J Neurol Sci. 2009;277(Suppl 1):S37-S41. doi:10.1016/S0022-510X (09)70011-5

47. Barin L, Salmen A, Disanto G, et al. The disease burden of multiple sclerosis from the individual and population perspective: which symptoms matter most? Mult Scler Relat Disord. 2018;25:112-121. doi:10.1016/j.msard.2018.07.013

48. Jongen PJ. Health-related quality of life in patients with multiple sclerosis: impact of disease-modifying drugs. CNS Drugs. 2017;31 (7):585-602. doi:10.1007/s40263-017-0444-x

\section{Publish your work in this journal}

Patient Preference and Adherence is an international, peer-reviewed, open access journal that focuses on the growing importance of patient preference and adherence throughout the therapeutic continuum. Patient satisfaction, acceptability, quality of life, compliance, persistence and their role in developing new therapeutic modalities and compounds to optimize clinical outcomes for existing disease states are major areas of interest for the journal. This journal has been accepted for indexing on PubMed Central. The manuscript management system is completely online and includes a very quick and fair peer-review system, which is all easy to use. Visit http:// www.dovepress.com/testimonials.php to read real quotes from published authors. 